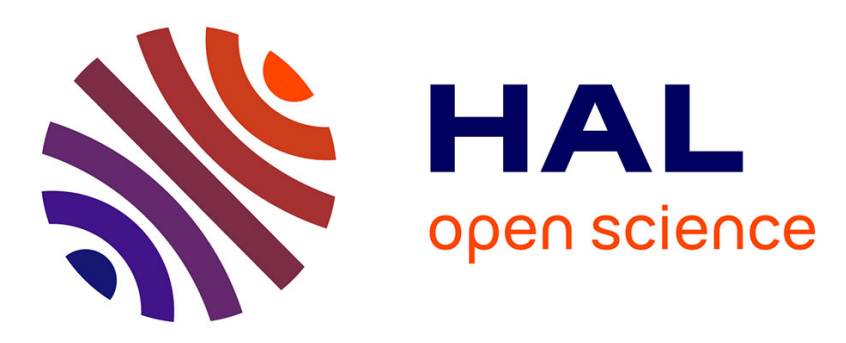

\title{
Contextualiser les pratiques sociolinguistiques : cheminements vers l'interprétation critique
}

\author{
Aude Etrillard
}

\section{To cite this version:}

Aude Etrillard. Contextualiser les pratiques sociolinguistiques : cheminements vers l'interprétation critique. Cahiers Internationaux de Sociolinguistique, 2014, Pluralité linguistique et culturelle : Actualité de la recherche en sociolinguistique, 1 (5), pp.135-145. 10.3917/cisl.1401.0135 . halshs-01397571

\section{HAL Id: halshs-01397571 \\ https://shs.hal.science/halshs-01397571}

Submitted on 16 Nov 2016

HAL is a multi-disciplinary open access archive for the deposit and dissemination of scientific research documents, whether they are published or not. The documents may come from teaching and research institutions in France or abroad, or from public or private research centers.
L'archive ouverte pluridisciplinaire HAL, est destinée au dépôt et à la diffusion de documents scientifiques de niveau recherche, publiés ou non, émanant des établissements d'enseignement et de recherche français ou étrangers, des laboratoires publics ou privés. 
Version «postprint» de l'article: Etrillard Aude, 2014, «Contextualiser les pratiques sociolinguistiques: cheminements vers l'interprétation critique", Cahiers internationaux de sociolinguistique, 2014, vol. $1, \mathrm{n}^{\mathrm{o}} 5$, p. 135-145.

\section{CONTEXTUALISER LES PRATIQUES SOCIOLINGUISTIQUES : CHEMINEMENTS VERS L'INTERPRETATION CRITIQUE. ${ }^{1}$}

Dans ce texte, je reviendrai sur mon orientation dans la pluralité des approches épistémologiques et méthodologiques des concepts de terrain et de contextualisation en sociolinguistique dans le cadre d'une recherche doctorale. Je montrerai les éléments qui auront permis une renégociation de ces concepts au cours de ma recherche. Cette narration de mes trajectoires convergentes, dans la discipline d'une part et sur mon terrain d'enquête d'autre part, me mènera à justifier du choix final d'une approche critique et interprétative dans le contexte spécifique de ma recherche, qui a pour objectif l'observation et l'analyse des modalités d'interactions entre des migrants britanniques et des individus autochtones dans des milieux ruraux en Bretagne.

\section{PREMIERES APPROCHES DU TERRAIN}

En 2007, dans le cadre d'une recherche de Master, je m'intéressai pour la première fois aux pratiques et représentations langagières de migrants britanniques installés dans les frontières administratives du Pays de Pontivy ${ }^{2}$, qui délimitaient alors un terrain. J'avais entendu certains membres de ma famille résidants en milieu rural en Bretagne discuter de l'installation massive " d'Anglais » autour de chez eux et, venant d'une licence d'anglais, mon « anglophilie» avait été piquée par des discours souvent peu valorisants concernant ces nouveaux migrants et leurs pratiques linguistiques, je réalisais qu'il y avait une opportunité pour une recherche en sociolinguistique, discipline dans laquelle je poursuivais ma formation. Au cours de cette recherche, j'ai découvert que les Britanniques formaient la plus grande population migrante en Bretagne (INSEE, 2008), qu'ils étaient majoritairement propriétaires de leurs habitations (Ibid.), que le phénomène migratoire se manifestait dans d'autres régions de France et d'Europe (Barou et Prado, 1995), et se concentrait dans des milieux ruraux en Bretagne, y intensifiant les pratiques anglophones. La rareté des références bibliographiques sur le sujet, au moment où j'ai commencé cette recherche, rendit mes premières entrées sur le terrain clairement empiriques. À partir d'une analyse sociolinguistique d'entretiens semi-directifs et d'observations participantes, il m'est apparu que les discours circulant sur le sujet étaient saturés de stéréotypes. On parlait donc des Anglais comme d'une catégorie homogène non francophone considérant qu'elle n'avait pas besoin de le devenir ; restant «entre eux» et «ne s'intégrant pas»; tirant profit d'une crise de l'immobilier, et parfois du système de santé français; ne faisant appel qu'à des artisans compatriotes pour leurs travaux de rénovation. Dans le même temps, ces discours étaient le plus souvent relativisés, déconstruits ou renégociés lorsqu'il s'agissait de sortir d'un imaginaire en décrivant des personnes et des situations précises. Les informateurs autochtones disaient également mobiliser leurs compétences anglophones occasionnellement. Par ailleurs, chez les élus locaux et d'autres acteurs du tissu social, on insistait sur l'opportunité d'une telle migration pour contrebalancer la désertification massive des territoires.

\footnotetext{
${ }^{1}$ Aude Etrillard, doctorante en Sciences du Langage, PREFics EA 4247 Université de Rennes 2, GIS Pluralités Linguistiques et Culturelles, aude.etrillard@gmail.com

${ }^{2}$ Une subdivision administrative du Département du Morbihan, rassemblant plusieurs communes. 
Du côté des informateurs britanniques rencontrés, je remarquais une forte volonté d'individuation par rapport à la catégorisation «Anglais»; mais aussi la reproduction de discours stéréotypés d'autochtones pour décrire certains de leurs compatriotes; une compétence communicationnelle souvent insuffisante pour comprendre et discuter avec les populations locales; une absence quasi totale de socialisation chez certaines personnes. L'expérience migratoire, la prise de contact avec les populations autochtones s'avéraient alors plus problématique qu'attendue et que décrite par les personnes interrogées. Beaucoup de ces problématiques semblaient se cristalliser autour des pratiques langagières.

Suite à cette recherche, un projet doctoral sembla pertinent afin d'approfondir les modalités des interactions au quotidien, entre migrants britanniques d'une part et avec les populations autochtones d'autre part, dans des milieux ruraux en Région Bretagne. Dans l'élaboration de ce nouveau projet, l'approche privilégiée concernant les concepts de terrain et de contexte, fut dans la continuité du premier :

- une approche co-constructiviste (Blanchet, 2001) impliquant que le corpus n'est pas un ensemble de données stables et objectives, mais le fruit d'une collaboration entre enquêtrice et «enquêté-e-s», dans la production de sens, au moment de l'entretien. (Ibid., Calvet et Dumont, 1999)

- une ethnographie de la communication, des pratiques langagières, interactionnelles et discursives, impliquant leur contextualisation afin de mettre en avant la prise en compte de ce contexte par les interactants dans leurs activités communicationnelles sociales, y comprit donc chez l'enquêtrice (Ibid., 101). Ceci m'amène à intégrer le terrain (puisque c'est sur le terrain qu'est produit le corpus) dans le concept de contexte, à l'instar de Feussi $(2006,158)$. Ceci implique par ailleurs la mise en relief de la subjectivité de la chercheure dans son processus de recherche, jusqu'à l'écriture, par exemple par l'emploi de la première personne, responsabilisant directement l'auteure face à ses propos.

- une démarche empirico-inductive (décrite, et non prescrite, chez Blanchet, 2001, 28), impliquant de ne pas «venir sur le terrain » avec des préconçus, mais devait rester ouverte à une interprétation émergeant empiriquement de l'analyse des interactions.

On peut voir dans cette approche une pratique tout à fait banale de l'enquête qualitative sociolinguistique, reposant sur «L'étude du langage ancrée dans ses conditions sociales de production - véritable pétition de principe de la sociolinguistique. » (Boutet et Heller, 2007, 306)

Pourtant, deux paradoxes apparaissent dans ma pratique de recherche au moment de présenter le travail de contextualisation, et donc au moment de découvrir mon terrain. En effet, «connaître son terrain » consistait initialement pour moi à rassembler un certain nombre de données démographiques, géographiques, et éventuellement historiques, pour proposer une description du terrain, alors délimité par des frontières administratives, non questionnées. La notion de contextualisation dans cette perspective s'en trouvait alors réduite à une liste d'éléments immédiatement observables dans le cadre de l'interaction et aux données disponibles pour objectiver le terrain. La dimension "sociale » des pratiques langagières, quant à elle, ne s'ancrait pas véritablement dans des théories plus transversales permettant de comprendre les dynamiques de l'environnement social. Si je convoquais quelques emprunts conceptuels à Bourdieu (1982) a posteriori pour appuyer théoriquement des observations, mon positionnement vis-à-vis du cadre plus large de la sociologie de Bourdieu n'était pas explicité pour autant. Au début du projet de recherche, la dimension sociale restait indéfinie, un élément allant de soi, qui serait donc forcément présent et visible dans mes entretiens et observations d'interactions de migrants et d'autochtones sur le terrain.

\section{PaRadoxes de la CONTEXTUalisation}

Pourquoi, si je me revendiquais d'une posture co-constructiviste, ne pas avoir exploré ma compréhension du « social »? J'y vois, d'une part l'héritage des premières formulations des théories sociolinguistiques constituées dans une opposition à la linguistique structurale (Gumperz et Hymes, 1972), et d'autre part l'apport de Goffman (1987 et Winkin, 2001) à la sociolinguistique. Si le " socio" de sociolinguistique signifie la volonté d'une prise en compte du langage en tant que « pratiques réelles », traversant et étant traversées par la socialisation, il n'est en effet pas problématisé 
plus avant chez de nombreux sociolinguistes, comme le soulignent Heller et Boutet $(2007,307)$, ou comme le reconnaissent Gumperz et Cook-Gumperz $(2008,538)$. La conséquence apparait de manière saillante, dans certains guides méthodologiques pour l'enquête, par exemple chez de Salins (1992) où l'on observe une approche méthodologique qui maintient la contextualisation dans un «ici et maintenant ». Par exemple la dimension émique de l'analyse de l'interaction (Salins de, 1992, 55) est restreinte au contexte formé par les seuls interactants, au moment de l'interaction, et non nécessairement, à son emboîtement plus large dans une dimension sociale, malgré une intention d'analyser "ces petites manifestations sociales qui participent à la forme sociale» (Ibid, 12). L'analyse de la compétence de communication, ce que Masquelier appelle "l'échafaudage» de Hymes (Masquelier, 78-79) est pris alors comme une fin en soi.

On trouve alors aussi dans ces pratiques l'approche d'Erving Goffman des interactions : puisque le social se construit dans l'activité interactionnelle, c'est par l'analyse de cette activité que l'on y accède. Chez Goffman, il s'agit même d'une posture assumée que de préférer aux "grandes théories » générales du social, qui ne sont pour lui pas véritablement aptes à éclairer la réalité de la vie sociale ordinaire, une analyse exclusivement basée sur l'observation des interactions qui cristallisent déjà l'ordre social (Winkin, 2001, 107). Si je poursuis le raisonnement, il s'agit donc de ne pas poser par principe l'existence d'une structure, mais de la faire émerger par l'analyse : l'interaction fait exister « son théâtre ".

Ceci implique il me semble, un choix entre deux options : soit l'on considère que « social » est ce que l'on observe, sans s'en expliquer plus que cela et en le contenant dans l'instantané de l'interaction; soit l'on considère que le social est dans un contexte visible et disponible au regard du chercheur, mais on refuse alors l'idée que toute entrée dans un terrain et donc la contextualisation est préalablement située et déjà orientée par des préjugés. L'effet, me semble-t-il, dans un sens comme dans l'autre, est de «brider » le travail interprétatif, en se privant des outils intellectuels permettant de construire une recherche. Et si le travail de contextualisation ne peut reposer sur cette dimension interprétative, le résultat ne peut être qu'une tentative d'objectivisation du contexte, et donc du terrain, dans une description d'éléments observables. Ainsi, alors même que j'aurai l'illusion de procéder de «bas en haut », de l'empirique vers l'analytique, je produis une inévitable objectivisation tacite, en niant l'existence de la subjectivité de mon interprétation préexistante du monde social.

Bien que je ne le pointe pas véritablement pour en venir aux mêmes propositions, Robillard souligne aussi ce paradoxe dans certains travaux en sociolinguistique: "une mise en scène objectivante et déresponsabilisante est réalisée, séparant fictivement les interprétations de corpus et les interprétations de contexte, et en donnant aux interprétations de contexte un statut factuel dont est censée découler immanquablement l'interprétation du corpus, laissant le chercheur enregistrer ce sens simplement. » (Robillard, D. de, 2011, 7).

Par ailleurs, Boutet et Heller soulignent qu'avec cette absence de problématisation du « social » "les analyses restent de nature corrélationniste où langagier et social sont liés par des manières de cooccurer ensemble » (Boutet et Heller, 2007, 307) comme je l'illustrai plus haut avec mon utilisation sporadique des concepts bourdieusiens.

Pourtant, Masquelier montre que chez Hymes, dès 1971, le projet est aussi d'intégrer l'analyse des activités de communication à une pensée d'un contexte social et politique (Masquelier, 2011, 80). Aussi lit-on chez Gumperz que le projet de la sociolinguistique interactionnelle est de "trouver des cas typiques de situations clés ou d'évènements de langage significatifs pour l'analyse de l'arrièreplan social et ethnographique. » (Gumperz, 1989, 15). On trouve également chez l'auteur les prémisses d'une approche critique en sociolinguistique : "Nous devons articuler une explication en termes politiques et institutionnels à une perspective centrée sur la micro-analyse de la pratique [...] quotidienne » (Ibid. pp 112-113).

Je note enfin qu'en 1972, dans sa contribution à Directions in Sociolinguistics, Bernstein, en maintenant ses ancrages sociologiques, "montre que la sociolinguistique peut et doit s'accrocher dans les théories sociales générales " (Bernstein, dans Gumperz et Hymes, 1972, 465) et, "si (...) les systèmes de parole sont générés, ou contrôlés, par des formes de relations sociales, alors les 
découvertes, aussi fascinantes et bien décrites soient-elles, ne peuvent être expliquées sans une théorie des relations sociales » (Ibid, pp 465-466).

\section{POUR UNE CONTEXTUALISATION INTERPRETATIVE}

$\mathrm{Au}$ fur et à mesure de mon travail de recherche, mes perspectives initiales sur le terrain et le contexte se sont révélées de plus en plus problématiques. D'abord, je ne pouvais pas ignorer le fait qu'en amont de mes analyses, $\mathrm{j}$ 'avais une interprétation, alors plus informée par mes convictions politiques que par des théories sociologiques, d'un monde social organisé par la reproduction d'inégalités entre les individus qui le constituent. En ceci, la sociolinguistique critique que propose Heller (2002) faisait fortement écho à ma posture, et il était difficilement concevable de faire une croix sur ce positionnement dans mon travail d'analyse.

Ensuite, il me semblait être arrivé à épuisement de ma grille d'analyse : il m'était possible de décrire les diverses formes interactionnelles que j'avais observées. Je percevais les diverses stratégies interactionnelles que les informateurs développaient, pour se différencier, se lier, se représenter, s'identifier, se valoriser, etc. Je pouvais voir que certaines étaient plus efficaces que d'autres, et en trouvais souvent les raisons dans le parcours de vie des informateurs et informatrices. On pourrait alors considérer que j'avais ainsi atteint le but initial de ma recherche. Néanmoins, ces analyses, se restreignant à une analyse "micro", contribuaient faiblement à produire une compréhension du phénomène migratoire que j'observais. Non que j'eusse peur de produire une ethnographie incomplète, puisqu'elle le serait nécessairement. Mais cela me mettait en porte à faux avec la posture interprétative (Blanchet, 2000) à laquelle j'avais l'ambition de contribuer dans mon travail. En effet le but était «de construire une interprétation modélisée, transférable et vérifiable, de phénomènes observés dans leurs fonctionnements complexes en contexte » (Ibid., 60).

Aussi, interpellée par plusieurs enquêté(e)s autochtones, je rencontrai les limites de mon approche :

Nadine $e^{4}$ : vous vous rendez compte c'est quand même euh un p'tit pat'lain de rien du tout/y a strictement RIEN/ nous on comprend pas POURquoi des Anglais viendraient viennent ICI franchement/

À Nadine, comme à d'autres, je reproduisais, face à ces interrogations, le discours des Britanniques interrogés à ce sujet : les conditions économiques ont rendu possible la recherche du calme, d'un autre rythme de vie, d'espace. Les informateurs et informatrices semblaient peu satisfait-e-s par ce discours, qu'ils et elles connaissaient déjà bien par ailleurs, et qui moins qu'une interprétation, consistait en une description des phénomènes qui ne faisait pas forcément sens pour ces derniers. Par ailleurs, les "facteurs » économiques ne semblaient que maigrement justifier la migration: tous ces facteurs existaient déjà depuis longtemps, en Centre Bretagne ou ailleurs, et influençaient l'intensité du phénomène, plutôt que ne l'expliquaient et je n'avais donc fait qu'une corrélation entre «des données ».

Un second événement vint faire écho à ces réflexions. Grâce à une énième reconfiguration de mes veilles documentaires, j'ai pu découvrir un rassemblement de chercheur-e-s autour de mes thématiques: les migrations de populations occidentales dans des pays étrangers, dans des zones de

\footnotetext{
${ }^{3}$ Mes traduction de l'anglais : « Bernstein shows that sociolinguistics can and must come to grips with general social theory » et «If, however, as Bernstein maintains, speech systems are generated, or controlled, by forms of social relations, then discoveries, however fascinating and well described, cannot be explained without a theory that embraces social relations. "

${ }^{4}$ Nadine (le nom a été changé) est une autochtone très active dans le réseau associatif d'une des communes étudiées dans cette recherche. Elle est une des personnalités médiatrices rencontrées permettant la mise en lien de Britanniques avec des autochtones.
} 
tourismes, et entre autres dans des zones rurales ${ }^{5}$. Parmi ces chercheur-e-s, Ferbrache (2011) qui observe ce phénomène migratoire à la lumière du contexte de globalisation. Et, quoique ne problématisant pas les contacts entre les populations et la dimension langagière de l'expérience migratoire, son travail m'apporte un éclairage essentiel sur les migrations britanniques générant un espace transnational, et illustrant l'émergence d'une société de réseau théorisée par Castells (2000). L'un des éléments définitoires de cette société est sa dynamique sur deux logiques spatiales, l'une émergente, dominant l'autre et organisée en flux (de population, de capitaux, d'information, de donnée, etc.) ; l'autre organisée dans une compréhension plus traditionnelle de l'espace social en lieu ${ }^{6}$ (Ibid., 378).

Enfin, c'est à la lecture des sixième et septième chapitres de Paths to Post-Nationalism de Heller (2011). En faisant le compte-rendu d'un effet de la globalisation sur un espace francophone rural au Canada, l'auteure nous présente extensivement le phénomène de commodification. Dans le cadre d'un processus de «développement» de ces régions autour de l'industrie touristique, Heller observe une objectivisation des éléments d'une identité sociolinguistique, pour renforcer l'image d'un territoire " authentique », rural et francophone, par opposition à "moderne», urbain et anglophone. Ces éléments sociolinguistiques deviennent alors une "valeur ajoutée » (Ibid, 124) des éléments exploitables pour se distinguer sur le marché touristique (Ibid, 128). Et, dans le même temps que des pratiques francophones sont utilisées pour faire la démonstration de cette identité authentique, les pratiques anglophones permettent une communication orientée vers les touristes/consommateurs anglophones (Ibid. 140), et d'engager une coopération économique pour développer le territoire. Se dessinent alors de multiples ambivalences, entre un désir de maintenir une identité authentique en contraste avec un monde global, et le désir de trouver sa place économique dans cette globalisation par le marché touristique; entre un désir de penser les pratiques langagières comme des éléments d'une authenticité identitaire, ou comme des compétences nécessaires pour une compétitivité sur le marché.

Les constats de Heller entrent alors inévitablement en écho avec mon terrain et me permettent d'esquisser un début de réponse aux interrogations des autochtones interviewés, mais également de trouver les pistes pour interpréter les éléments apparemment paradoxaux que je rencontre : d'un côté la recherche d'une idylle rurale (Benson, 2011) authentique des migrants britanniques, arrivés pour la plupart suite à une expérience touristique, et de l'autre un certain désenchantement dans l'expérience de « la vie rurale ». D'un côté un discours dans la population autochtone trahissant l'anxiété de voir le territoire s'appauvrir et rester en marge de la modernité, et de l'autre la crainte de se voir désapproprier le territoire par une logique de marché. Cette perspective conduit à penser la répartition des ressources sociolinguistique dans le contexte de la globalisation: "ce qui importe dans la "globalisation", ce sont les façons dont les marchés locaux et régionaux sont intégrés dans des marchés globaux, via des régimes de régulation basés sur la coopération économique plutôt que sur un colonialisme" (Heller, 2011, 20). Et, " le langage n'est pas une fenêtre transparente rendant visible les processus sociaux, mais plutôt un élément constitutif de ces derniers » (Ibid, ,49) ${ }^{7}$

Dès lors, j'ai fait le choix interprétatif de voir mon terrain non seulement comme le site de mes entretiens et observations, mais comme un processus social, un phénomène migratoire, une manifestation de la globalisation qui repose sur la répartition inégale des ressources - notamment langagières - mobilisées par les individus dans la production d'un ordre social. Un phénomène qui

\footnotetext{
${ }^{5}$ Le groupe se nomme Lifestyle Migration, après les travaux de Benson et O’Reilly (2009). Pour une bibliographie, voir http://www.uta.fi/yky/lifestylemigration/index.html.

${ }^{6}$ Spaces as flows et spaces as places (Castells, 2000, 376)

${ }^{7}$ Mes traductions de 1'anglais : « what is important about « globalization » is the ways in which local and regional markets are integrated into global ones via regimes of regulation based on economic cooperation rather than colonialism » et " Language is not a transparent window into social processes but, rather, a constitutive element of them $»$.
} 
met donc en lien et hiérarchise des espaces globaux et des espaces locaux, et qui amène les individus à s'y positionner. Ceci m'a conduite par exemple à observer les fonctions politiques et économiques des pratiques anglophones sur le terrain (Etrillard, en cours ${ }^{\mathrm{a}}$ ), les enjeux linguistiques dans la constitution d'une image attractive du territoire (Etrillard, en cours ${ }^{c}$ ), ou encore les variations des conditions de "l'intégration» et de l'accueil des populations étrangères, notamment par la hiérarchisation des catégories sociolangagières (Etrillard, en cours ${ }^{b}$ et en cours ${ }^{\mathrm{d}}$ ).

Les conséquences de ce choix interprétatif sur ma perception des phénomènes, et des pratiques interactionnelles que j'observe sont alors grandes et me permettent de réinterpréter les enjeux sociaux de la place accordée ou négociée des pratiques identifiées comme anglophones sur mon terrain. On dépasse alors une dialogique micro-macro pour rejoindre une approche complexe (Morin, 1996) de la dialectique, soit du lien non hiérarchisé et inextricable, et parfois paradoxal des pratiques interindividuelles et des dynamiques sociétales. Ceci me permet aussi d'interpréter les migrations britanniques comme émergeant d'un contexte social propre à cette fin de XXe siècle et début de vingtet-unième, favorisées par la montée d'une vie sociale de " réseau » (Castells, 2000), le développement des télécommunications, l'individuation du sujet réflexif (Benson et O'Reilly, 2009, 3), le développement des compagnies aériennes à bas coût, le marketing touristique, la circulation facilitée des flux de capitaux, l'internationalisation des transactions immobilières, conséquences des stratégies des agences immobilières elle-même, la flexibilité des conditions de travail, etc.

En renégociant cette interprétation du terrain de ma recherche, il me semble alors avoir opéré le retournement $\mathrm{du}$ « sablier », pour reprendre l'image de Philippe Blanchet $(2000,40)$ : «La démarche va du global (prise d'indices multiples en contexte complexe par observation participante) à l'analytique [...] pour revenir à une synthèse interprétative» (Ibid., 41). Ce va-et-vient, inductif/déductif tel que préconisé par Blanchet (Ibid., 32), de l'étude de cas au global, du global à l'étude de cas, fait d'ailleurs particulièrement sens dans le cadre interprétatif de la globalisation : un phénomène dont les formes plurielles se déclinent, en fonction des contextes où il se manifeste. La diversité des pratiques sociales et linguistiques peut alors être mise en lien dans un cadrage sociologique laissant place à la fois à la pluralité des expériences interactionnelles et à la mise en relief de processus sociaux organisateurs tels que la répartition et la concentration des ressources, produit et en partis producteurs de ces diversités.

En rappelant que dans un cadre interprétatif le travail d'analyse « renvoie ainsi a une vision et a une connaissance globale des fonctionnements sociolinguistiques, laquelle fait également appel à des données macro-sociolinguistique », Blanchet (ibid., 59), incite à se positionner dans des théories sociales. Étant les produits d'une histoire, de cultures, d'interactions, de pratiques et de nos représentations, et en étant processuels, nos terrains sont des éléments à interpréter dans une sociologie ou le micro et le macro sont intégrés. Cette posture me permet alors d'assumer ma subjectivité dès le travail de contextualisation et de constitution du terrain, ainsi que de référencer mes interprétations aux théories critiques, dissolvant le paradoxe de ma compréhension initiale du concept de terrain. Enfin, en guise d'hommage, je soulignerai que cette approche me permet de m'inscrire dans le projet initial de Gumperz cité plus haut, que je tenterai donc de prolonger dans mon travail de thèse.

\section{BibliographiE}

BENSON M. et O'REILLY, 2009, Lifestyle Migration, Ashgate, Farnham, 168 pages.

BENSON M., 2011, The British in Rural France, Manchester University Press, Manchester, 182 pages.

BLANCHET P., 2000, Linguistique de Terrain, Presses Universitaires de Rennes, Rennes, 195 pages.

BOUTET J. et HELLER M., «Enjeux sociaux de la sociolinguistique : pour une sociolinguistique critique », Langage et société, 2007/3 n 121-122, p. 305-318.

CALVET L.-J. et DUMONT P. (dir.), 1999, L'enquête sociolinguistique, L'Harmattan, Paris, 190 pages.

CASTELLS M., 2000, The Rise of The Network Society: The Information Age: Economy, Society and Culture, Wiley-Blackwell, Oxford, 594 pages. 
ETRILLARD A., (En cours ${ }^{\text {a }}$ ), "English Spoken. Une perspective critique sur les pratiques anglophones en milieu rural breton ", Actes du congrès du Réseau Francophone de Sociolinguistique 2013. Les locuteurs et les langues : pouvoirs, non-pouvoirs et contre-pouvoirs. 3-5 juillet 2013.

ETRILLARD A., $\left(\right.$ En cours ${ }^{b}$ ), «Les migrants britanniques en Bretagne intérieure : représentations de l'espace social et stratégies interactionnelles », publication aux Presses Universitaires de Rennes dans le cadre du cycle de séminaire Identités, migrations, diasporas de Équipe de recherche sur les minorités nationales et les ethnicités.

ETRILLARD A., (En cours ${ }^{\mathrm{c}}$ ), « From desire to experience and back again : sociolinguistic trajectories of British migrants in rural Brittany », Communication au Sociolinguistics Symposium 20. 15-18 Juin 2014.

ETRILLARD A., $\left(\right.$ En cours ${ }^{\mathrm{d}}$ ), « Towards a critical and ethnographic approach to language practices in lifestyle migrations », dans Torkington K., Sardinha J, et David I. (éds), Practising the Good Life/ The Good Life in Practice, Ashgate, Farnham.

FERBRACHE F., 2011, Transnational spaces within the European Union: the geographies of British migrants in France, Thèse de Doctorat, Université de Plymouth, Plymouth, 308 pages.

FEUSSI V. 2006, Une construction du français à Douala - Cameroun, Thèse de Doctorat, Université François Rabelais de Tours, Tours, 717 pages.

GOFFMAN E., 1987, Les rites d'interaction, Editions de Minuit, Paris, 240 pages.

GUMPERZ J.J et COOK-GUMPERZ J., 2008, "Studying language, culture, and society: Sociolinguistics or linguistic anthropology? », Journal of Sociolinguistics 12/4, 2008, pp. 532-545.

GUMPERZ J.J. and HYMES D. (ed.), 1972, The Ethnography of Communication - Directions in Sociolinguistics, Holt, Rinehart and Winston, New-York, 598 pages.

HELLER M., 2002, Éléments d'une sociolinguistique critique, Didier, Paris, 175 pages.

HELLER M., 2011, Paths to Post-Nationalism, Oxford University Press, New-York, 223 pages.

INSEE, 2008, « Peu d'immigrés en Bretagne mais toujours plus de Britanniques », Octant $\mathrm{n}^{\circ} 112$, url : http://www.insee.fr/fr/INSEE_REGIONS/Bretagne/rfc/docs/oc112art3.pdf, mise en ligne : avril 2008, 259 Ko.

MASQUELIER B., 2011, "Anthropologie sociale et analyse du discours », Langage et société, 2005/4 n 114 , p. 73-89.

MORIN E., 1990, Introduction à la pensée complexe, Éditions du Seuil, Paris, 158 pages.

ROBILLARD de, D. "Contribution au débat du Réseau francophone de sociolinguistique: les "langues-cultures" sont-elles neutres dans le cadre d'une recherche, particulièrement francophone?", $11 \mathrm{p}$.

SALINS de. G.-D., 1992, Une introduction à l'ethnographie de la communication : pour la formation à l'enseignement du français langue, Didier, Paris, 223 pages.

WINKIN Y., 2001, Anthropologie de la Communication, Éditions du Seuil, Paris, 332 pages. 